\title{
Reviving Cell-Based Regenerative Medicine for Heart Reconstitution with Efficiency in Deriving Cardiac Elements from Pluripotent Human Embryonic Stem Cells
}

\author{
Xuejun H Parsons ${ }^{1,2 *}$ \\ ${ }^{1}$ San Diego Regenerative Medicine Institute, San Diego, CA 92109, USA \\ ${ }^{2}$ Xcelthera, San Diego, CA 92109, USA
}

To regrow the human heart had only been a dream, not a possibility before the derivation of human embryonic stem cells (hESC) from the inner cell mass (ICM) or epiblast of the human blastocyst [1,2]. In the adult heart, cardiomyocytes, the mature contracting cardiac muscle cells, are terminally differentiated and unable to divide. There is no evidence that stem/precursor/progenitor cells derived from mature tissues, such as bone marrow, cord blood, umbilical cord, mesenchymal stem cells, patients heart tissue, placenta, or fat tissue, are able to give rise to the contractile heart muscle cells following transplantation into the heart $[1,2]$. Despite numerous reports about cell populations expressing stem/precursor/progenitor cell markers identified in the adult hearts, the minuscule quantities and growing evidences indicating that they are not genuine heart cells and that they give rise predominantly to smooth muscle cells rather than functional cardiomyocytes have diminished any enthusiasm [1-3]. Therefore, there is mounting skepticism about the existence of endogenous human cardiac stem cells after birth. Derivation of hESCs in vitro from the IVF leftover embryos has brought a new era of cellular medicine for the heart $[1,2]$. The intrinsic ability of a hESC to both unlimited self-renew and differentiation into cardiac elements makes it a practically inexhaustible source of replacement cells for the damaged heart. The hESC cardiac cell therapy derivatives are emerging as a new type of pharmacologic agent of cellular entity in cellbased regenerative medicine because they have direct pharmacologic utility and capacity for human myocardial tissue reconstitution and contractile function restoration that the conventional compound drug of molecular entity lacks [1-6]. The pharmacologic activity of human cardiac stem cells is measured by their extraordinary cellular ability to regenerate the functional and structural cardiac tissue element, thus, the contractile cardiomyocytes that has been damaged or lost. Therefore, the pharmacologic utility of human cardiac stem cells cannot be satisfied only by their chaperone activity, if any, to differentiate into nonfunctional smooth muscle cells or endothelial cells, or produce trophic or protective molecules to rescue endogenous dying cardiomyocytes that can simply be achieved by a compound drug of molecular entity. Although a vast sum of government and private funding has been spent on looking for adult alternates, such as reprogramming and trans-differentiation of fibroblasts or mature tissues, so far, only human cardiac stem/precursor/progenitor cells derived from embryooriginated hESCs have shown such cellular pharmacologic utility and capacity adequate for myocardium regeneration in pharmaceutical development of stem cell therapy for the damaged heart [1-6].

Due to the prevalence of cardiovascular disease worldwide and acute shortage of donor organs or adequate human myocardial grafts, there is intense interest in developing hESC-based therapy for heart disease and failure [1,2]. The hESCs and their derivatives are considerably less immunogenic than adult tissues [1-3]. It is also possible to bank large numbers of human leukocyte antigen isotyped hESC lines so as to improve the likelihood of a close match [1-3]. However, the heart does not just grow out of hESCs in a petri dish. One of the major challenges in developing hESC therapies for cardiac repair is to determine the necessary molecular and cellular cues that direct efficient cardiac lineage-specific differentiation of pluripotent hESCs. The normal human developmental pathways that generate cardiomyocytes remain poorly understood. As a result, directing hESC differentiation towards cardiomyocytes in a systematic manner has proved difficult. Only a very small fraction of pluripotent hESCs differentiate into cardiomyocytes through spontaneous germ-layer induction in culture $[1,2]$. Although such hESC-derived immature cardiomyocytes can be enriched to attenuate the progression of heart failure in acute myocardial infarction model, the grafts generated by cell transplantation have been small and insufficient to restore heart function or to alter adverse remodeling of chronic infarcted models following transplantation $[1,2]$. Functional enhancement in preclinical animal models by such hESC-derived cardiomyocytes through conventional multi-lineage germ-layer induction has been limited to mid-term at most, equivalent to perhaps a few months in humans, and there is no evidence that the underlying mechanism depends on the contractile properties of the transplanted human cells [1,2]. Growing evidences indicate that incomplete lineage specification of pluripotent cells often result in poor performance of such stem cell derivatives and/or tissueengineering constructs following transplantation [1,2]. In addition, the need for foreign biologics, such as animal feeder cells and proteins, for derivation, maintenance, and differentiation of hESCs may make direct use of such cells and their derivatives in patients problematic $[1,2]$.

Clinical applications of hESC cardiac cell therapy derivatives provide the right alternative for heart disease and failure that the conventional compound drug of molecular entity cannot. Recent advances and technology breakthroughs in hESC research have overcome some major obstacles in bringing hESC therapy derivatives towards clinical applications, including resolving minimal essential human requirements for de novo derivation of clinically-suitable stable hESC lines and direct conversion of such pluripotent hESCs into a large supply of clinical-grade functional human neuronal or cardiomyocyte cell therapy products to be translated to patients for CNS or heart repair [1,2,4-12]. Without an understanding of the essential developmental components for sustaining hESC pluripotence and selfrenewal, hESC lines are at risk for becoming unhealthy and unstable after prolonged culturing under animal feeders, feeder-conditioned

${ }^{*}$ Corresponding author: Xuejun H Parsons, San Diego Regenerative Medicine Institute, San Diego, CA 92109, USA, E-mail: parsons@SDRMI.org

Received June 18, 2013; Accepted June 18, 2013; Published June 21, 2013

Citation: Parsons XH (2013) Reviving Cell-Based Regenerative Medicine for Heart Reconstitution with Efficiency in Deriving Cardiac Elements from Pluripotent Human Embryonic Stem Cells. Cardiol Pharmacol 2: e112. doi:10.4172/2329-6607.1000e112

Copyright: (c) 2013 Parsons XH. This is an open-access article distributed under the terms of the Creative Commons Attribution License, which permits unrestricted use, distribution, and reproduction in any medium, provided the original author and source are credited. 
Citation: Parsons XH (2013) Reviving Cell-Based Regenerative Medicine for Heart Reconstitution with Efficiency in Deriving Cardiac Elements from Pluripotent Human Embryonic Stem Cells. Cardiol Pharmacol 2: e112. doi:10.4172/2329-6607.1000e112

media, or artificially-formulated chemically-defined conditions $[1,5,13]$. Resolving minimal essential human requirements for sustaining embryonic pluripotence allows all poorly-characterized and unspecified biological components and substrates in the culture system, including those derived from animals, to be removed, substituted, and optimized with defined human alternatives for de novo derivation and long-term maintenance of GMP-quality xeno-free stable hESC lines and their human cell therapy derivatives $[1,5]$. Formulation of minimal essential defined conditions renders pluripotent hESCs be directly and uniformly converted into a specific neural or cardiac lineage by small signal molecule induction [1-12]. Such milestone advances and medical innovations in hESC research enable high efficient generation of a large supply of high purity clinical-grade hESC neuronal and heart muscle cell therapy products as powerful cellular medicines that offer adequate pharmacologic utility and capacity for CNS and heart regeneration in the clinical setting that no conventional drug of molecular entity can. For end-stage heart failure, stem-cell-mediated cellular regenerative approach cannot be used as an alternative approach to heart transplantation. Therefore, developing the technology of using hESC-derived cardiac elements to reconstitute human hearts in vitro as the replacement organ could provide alternative treatment options to donor-based heart transplantation [1,2]. The relative early stage and simplicity in development and maturation of the embryonic heart makes it also possible to be the first organ to be reconstituted from hESCs, the in vitro representation of the ICM/epiblast from which the heart is formed as the first organ. Establishing a controllable differentiation route to efficiently generate a large supply of human cardiac elements from hESCs makes it become feasible to reconstitute the human beating heart with human contractile heart muscle and the cardiovascular architecture of intact 3D geometry and vasculature of the whole heart. Transforming pluripotent hESCs into fate-restricted cardiac cell therapy derivatives dramatically increases the clinical efficacy of graft-dependent repair and safety of hESC-derived cellular products, bringing cell-based regenerative medicine to a turning point for heart disease and failure.

\section{References}

1. Parsons XH, Teng YD, Moore DA, Snyder EY (2011) Patents on technologies of human tissue and organ regeneration from pluripotent human embryonic stem cells. Rec Pat Regen Med 1: 142-163.

2. Parsons XH (2012) Mending the broken heart - Towards clinical application of human embryonic stem cell therapy derivatives. J Clin Exp Cardiolog 3: e116.

3. Passier R, van Laake LW, Mummery CL (2008) Stem-cell-based therapy and lessons from the heart. Nature 453: 322-329.

4. Parsons $\mathrm{XH}$, Teng YD, Parsons JF, Snyder EY, Smotrich DB, et al. (2011) Efficient derivation of human cardiac precursors and cardiomyocytes from pluripotent human embryonic stem cells with small molecule induction. J Vis Exp e3274.

5. Parsons JF, Smotrich DB, Gonzalez R, Snyder EY, Moore DA, et al. (2012) Defining conditions for sustaining epiblast pluripotence enables direct induction of clinically-suitable human myocardial grafts from biologics-free human embryonic stem cells. J Clin Exp Cardiolog S9: 001.

6. Parsons XH (2012) MicroRNA profiling reveals distinct mechanisms governing cardiac and neural lineage-specification of pluripotent human embryonic stem cells. J Stem Cell Res Ther 2: 124.

7. Parsons XH (2012) The dynamics of global chromatin remodeling are pivotal for tracking the normal pluripotency of human embryonic stem cells. Anat Physiol S3: 002.

8. Parsons XH, Teng YD, Parsons JF, Snyder EY, Smotrich DB, et al. (2011) Efficient derivation of human neuronal progenitors and neurons from pluripotent human embryonic stem cells with small molecule induction. J Vis Exp 56: e3273.

9. Parsons $\mathrm{XH}$ (2012) An engraftable human embryonic stem cell neurona lineage-specific derivative retains embryonic chromatin plasticity for scale-up CNS regeneration. J Regen Med Tissue Eng 1: 3.

10. Parsons XH, Parsons JF, Moore DA (2012) Genome-scale mapping of microRNA signatures in human embryonic stem cell neurogenesis. Mol Med Ther 1: 105

11. Parsons XH (2013) Human stem cell derivatives retain more open epigenomic landscape when derived from pluripotent cells than from tissues. J Regen Med 1: 2 .

12. Parsons $\mathrm{XH}$ (2013) Embedding the future of regenerative medicine into the open epigenomic landscape of pluripotent human embryonic stem cells. Annual Review \& Research in Biology 323-349.

13. Amps K, Andrews PW, Anyfantis G, Armstrong L, Avery S, et al. (2011) Screening ethnically diverse human embryonic stem cells identify a chromosome 20 minimal amplicon conferring growth advantage. Nat Biotechnol 29: 1132-1144. 